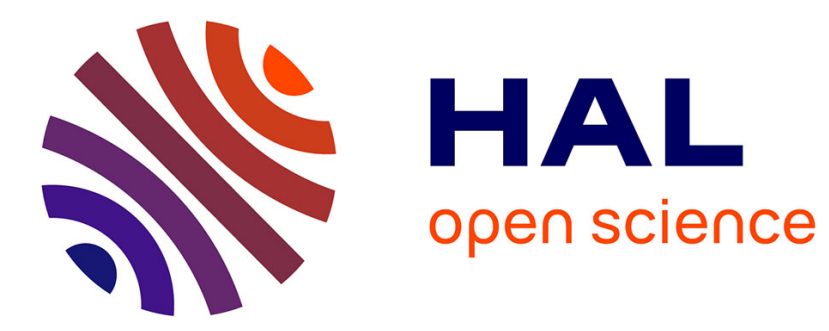

\title{
Structure and dynamics of dense water-in-oil microemulsions below percolation threshold
}

Si Chen, C. Ku, J. Rouch, P. Tartaglia, C. Cametti, J. Samseth

\section{To cite this version:}

Si Chen, C. Ku, J. Rouch, P. Tartaglia, C. Cametti, et al.. Structure and dynamics of dense water-inoil microemulsions below percolation threshold. Journal de Physique IV Proceedings, 1993, 03 (C1), pp.C1-143-C1-161. 10.1051/jp4:1993113 . jpa-00251556

\section{HAL Id: jpa-00251556 https://hal.science/jpa-00251556}

Submitted on 1 Jan 1993

HAL is a multi-disciplinary open access archive for the deposit and dissemination of scientific research documents, whether they are published or not. The documents may come from teaching and research institutions in France or abroad, or from public or private research centers.
L'archive ouverte pluridisciplinaire HAL, est destinée au dépôt et à la diffusion de documents scientifiques de niveau recherche, publiés ou non, émanant des établissements d'enseignement et de recherche français ou étrangers, des laboratoires publics ou privés. 


\title{
Structure and dynamics of dense water-in-oil microemulsions below percolation threshold
}

\author{
S.H. CHEN, C.Y. KU, J. ROUCH ${ }^{*}$, P. TARTAGLIA ${ }^{* *}$, C. CAMETTI ${ }^{* *}$ and J. SAMSETH ${ }^{* * *}$ \\ Department of Nuclear Engineering, 24-211, Massachusetts Institute of Technology, Cambridge, MA 02139, \\ U.S.A. \\ * CPMOH, Université Bordeaux I, 351 Cours de la Libération, 33405 Talence, France \\ "* Dipartimento di Fisica, Universita di Roma "La Sapienza", Piazzale Aldo Moro 2, 00185 Roma, Italy \\ *** Institut for Energiteknikk, P.O. Box 40, 2007 Kjeller, Norway
}

\begin{abstract}
Résumé
Le système de microémulsions AOT/eau/décane présente une phase intéressante au voisinage de la température ambiante. Le diagramme de phase (température-fraction volumique) a un point critique bas, correspondant à une fraction volumique d'environ $10 \%$, et est caractérisé par une ligne de percolation qui s'êtend du point critique jusqu'au grandes fractions volumiques. La phase obtenue est constituée de gouttelettes d'eau sphériques polydisperses entourées d'une monocouche d'AOT, et dispersées dans un continuum d'huile. Ces gouttelettes interagissent entre elles via une interaction de type "coeur dur" à laquelle s'ajoute une interaction à courte distance qui augmente avec la température. Nous montrons que le modèle de sphères de Baxter rend compte d'une maniēre quantitative de la structure de la phase observée ainsi que de la ligne de percolation pourvu que le paramètre d'adhérence des sphères soit une fonction convenable de la température. Afin de dēterminer la dépendance en température de ce paramètre, nous utilisons les facteurs de structure mesurés par diffusion de neutrons aux petits angles, au-dessous de la température critique. Nous étudions aussi la dynamique des gouttelettes au-dessous et à l'approche de la ligne de percolation par diffusion de lumiēre. En supposant l'existence de clusters fractales polydisperses, résultant de l'attraction des gouttelettes entre elles, nous pouvons calculer d'une maniëre quantitative le premier cumulant et la fonction de corrélation dépendant du temps de la densité de gouttelette. L'analyse des données de SANS indique que, au-dessus de la ligne de percolation, on passe graduellement d'une structure en clusters à une structure ordonnée bicontinue.
\end{abstract}

\begin{abstract}
The three-component ionic microemulsion system consisting of AOT/water/decane shows an interesting phase behavior in the vicinity of room temperature. The phase diagram in the temperature-volume fraction (of the dispersed phase) plane exhibits a lower consolute critical point around $10 \%$ volume fraction and a percolation line, cutting across the plane starting from the vicinity of the critical point, extends to high volume fraction side, at progressively lower temperatures. This phase behavior can be understood in terms of a system of polydispersed spherical water droplets, each coated by a monolayer of AOT, dispersed in a continuum of oil. These droplets interact with each other via a hard-core plus a short-range attractive interaction, the strength of which increases with temperature. We show that Baxter's sticky sphere model can account for the phase behavior including the percolation line, quantitatively, provided that the stickiness parameter is a suitable function of temperature. We use the structure factors measured by small angle neutron scattering below the critical temperature to determine this functional dependence. We also investigate the dynamics of droplets below and approaching the percolation line by dynamic light scattering. Both the first cumulant and the droplet density time correlation function can be quantitatively calculated by assuming the existence of polydispersed fractal clusters form by the microemulsion droplets due to the attraction. Analysis of SANS data indicates that above the percolation line, the clustered droplets structure gradually transforms into an ordered bicontinuous structure.
\end{abstract}




\section{Introduction}

The three-component ionic microemulsion system made of anionic surfactant, sodium di-2-ethylhexylsulfosuccinate (AOT), water and decane are rather unique among other popular microemulsion systems because, near room temperature, it does not show the well-known 2-3-1 phase progression, the well-known signature of a typical microemulsion system, as the surfactant concentration is increased from zero to more than $10 \%$. The reason is that around the room temperature the surfactant film, consisting of AOT molecules, possesses a spontaneous curvature toward water due to hydrophilicitylipophylicity imbalance of AOT molecules in this temperature range. Thus one finds in the ternary phase diagram a large one-phase region, called $\mathrm{L}_{2}$ phase, extending from the decane corner into the middle of the phase triangle. In the $\mathrm{L}_{2}$ phase the microemulsions consist of water doplets, coated by a monolayer of AOT, dispersed in decane. Previous SANS experiments verified that the average radius $\langle R\rangle$ of the water droplets is determined essentially by the molar ratio of water to AOT, called $W$, in the system. An approximate empirical relationship between the radius and $W$ is: $\langle R\rangle=(3 / 2) W$. Thus, for $W=40$., the average water droplet radius is about $60 \AA$ [1]. This water-in-oil droplet structure is maintained even if the volume fractions of water and oil are equal [2], provided the temperature is below 25 degrees centigrade. This case is in sharp contrast to the common situation that, for equal water and oil volume fractions, the microstructure of one-phase microemulsions were generally found to be bicontinuous [3,4]. Even for the AOT/water/decane system, when small amount of salt $(\mathrm{NaCl})$ is added, the common 2-3-1 phase progression is obtained at around the hydrophile-lipophile balance temperature of 40 degree centigrade [5] and a SANS experiment in the one-phase channel at this temperature conclusively showed that the microstructure is bicontinuous [6]. This unusual droplet structure in the ternary AOT/water/decane system can, however, be used to realize an interesting coexistence of a critical phenomenon at low volume fractions and high temperatures and a percolation phenomenon at high volume fractions and lower temperatures. In fact, this is a rare situation in which one can study, in a real system, the analogy, in both the static and dynamic properties, between approaching the critical point and percolation point from below. We shall exploit and stress this analogy throughout in this paper.

Figure 1 shows the T- $\phi$ phase diagram of $\mathrm{AOT} / \mathrm{H}_{2} \mathrm{O} /$ decane system when the water to AOT molar ratio $W=40.8$. Substitution of $\mathrm{H}_{2} \mathrm{O}$ by $\mathrm{D}_{2} \mathrm{O}$ will merely shift all the phase boundaries up by about 2 degrees. $\phi$ denotes the volume fraction of the dispersed phase, in this case the AOT plus water. In the diagram, one sees a one-phase $\left(\mathrm{L}_{2}\right)$ region below 40 degree centigrade. In the interval of $\phi$ between zero and 0.4 , there is a cloud point curve separating the one-phase droplet microemulsions from two-phase droplet microemulsions. A previous SANS experiment established that the average droplet sizes and their size distributions are within the experimental error identical in the one-phase and two-phase regions [1]. The critical volume fraction is approximately 0.1 and the critical temperature is 40 degrees [7] in $\mathrm{H}_{2} \mathrm{O}$. Above the volume fraction of 0.4 there is phase boundary between the $\mathrm{L}_{2}$ and a lamellar phase where the microstructure is ordered and bicontinuous in water and decane. The novelty of this phase diagram is, however, the existence of a percolation line, extending from the critical point all the way to higher volume fractions, gradually decreasing in temperature to about 23 degrees at $\phi=0.7$. Below the percolation line the microemulsion is non-conducting but above the percolation line it becomes conducting. In crossing the line, the conductivity increases by over five orders of magnitude. Figure 2 shows a set of logarithm of conductivities $\sigma$ as functions of $T$ and $\phi$. One sees clearly a set of steeply rising sigmoidal curves that can be used to define a set of loci $\left\{\mathrm{T}_{\mathrm{p}}, \phi_{\mathrm{p}}\right\}$ in terms of their inflection points. The asymptotic behavior of conductivity 


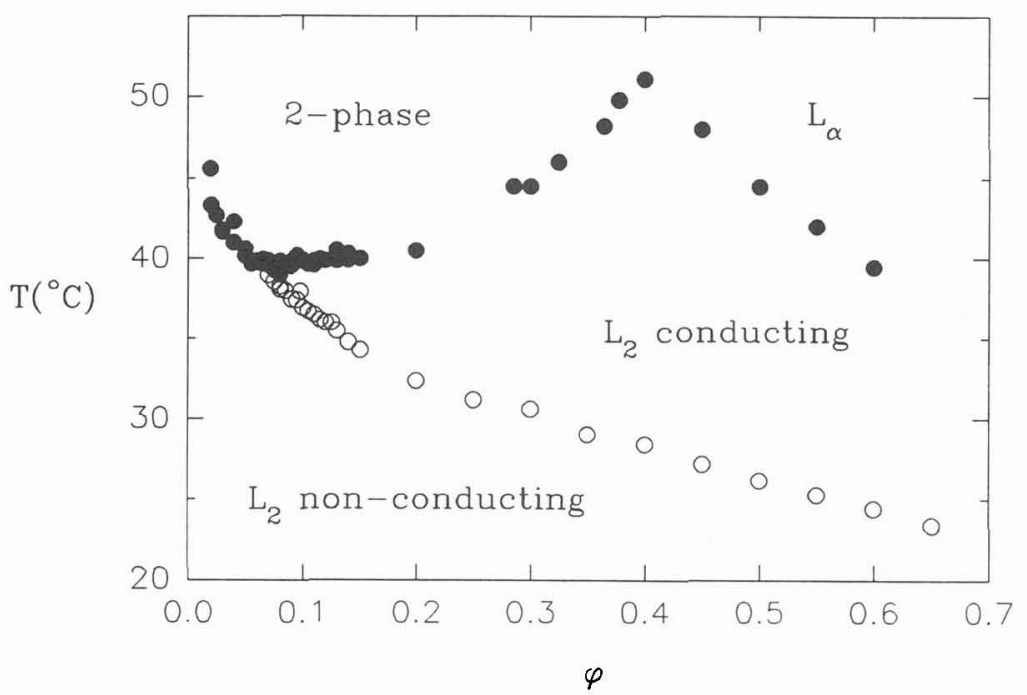

Fig. 1, Projection of the phase prism of $\mathrm{AOT} / \mathrm{H}_{2} \mathrm{O} /$ decane system, at $\mathrm{W}=40.8$ and one atmospheric pressure, on the temperature-volume fraction plane. The black circles are the experimental points [8].

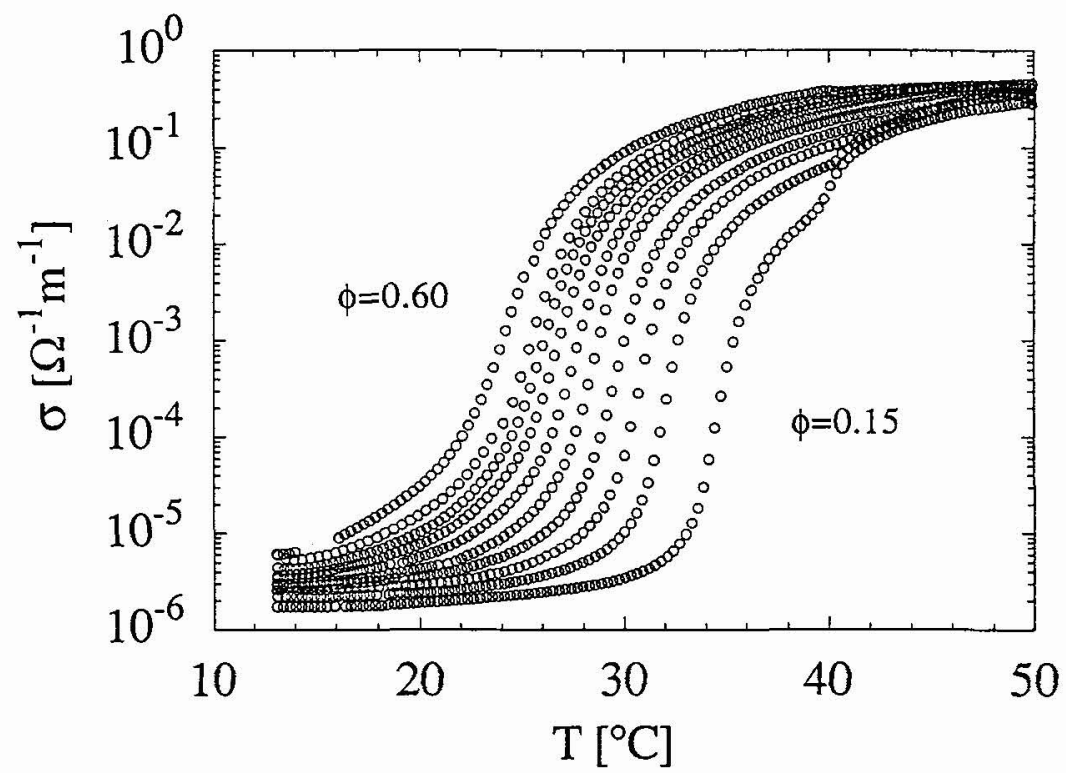

Fig. 2, DC conductivity of the $\mathrm{AOT} / \mathrm{H}_{2} \mathrm{O} /$ decane system as a function of volume fraction and temperature, showing the percolation behavior.

near the threshold, at a given $\phi$, can be expressed as:

$$
\sigma=A \cdot\left(\frac{T_{p}-T}{T_{p}}\right)^{-s^{\prime}}
$$


coming up from below, and

$$
\sigma=B \cdot\left(\frac{T-T_{p}}{T_{p}}\right)^{t}
$$

going down from above. The exponents $s^{\prime}$ and $t$ have been determined experimentally to be $1.2 \pm 0.1$ and $1.9 \pm 0.1$ respectively [8]. The exponents are the same when $T$ is fixed but $\phi$ is varied [8]. The exponent $s^{\prime}$, determined from conductivities below the threshold, agrees with the value of the index proposed in the so called dynamic percolation theory $[9,10]$, which is distinct from the standard static percolation exponent $s=0.73$ [11]. On the other hand, the exponent $t$ deduced from data above the threshold agrees with the static or geometric percolation theory [11]. In the theory of dynamic percolation, the conduction of electricity is conjectured to be mediated by charge carriers (presumably the sodium counterions from the AOT molecules) which migrate rapidly among microemulsion droplets forming transient fractal clusters, due to a short range attractive interaction between the droplets. The percolation threshold is defined theoretically to be a point where the average cluster size becomes infinity, namely, when at least one cluster spans the entire sample. Note, for this definition, finite conduction can occur already below the threshold because even there an infinite cluster can exist. Figure 3 depicts the asymptotic behavior of the conductivity by plotting scaled conductivities $T_{p}(\sigma / A)^{1 / s^{\prime}}$ and $T_{p}(\sigma / B)^{-1 / t}$ as a function of $\mathrm{T}-\mathrm{T}_{\mathrm{p}}$. It is seen that above the threshold, all the data at different volume fractions follow the asymptotic relation eq.(2) very well. However, below the threshold, the asymptotic relation eq.(1) holds better for higher volume fractions. For lower volume fractions, say below $\phi=0.4$, the range of validity $\Delta \mathrm{T}$ is very small. Thus experimentally the manifestation of dynamic percolation is observable only when $\phi$ is greater than about 0.45 .

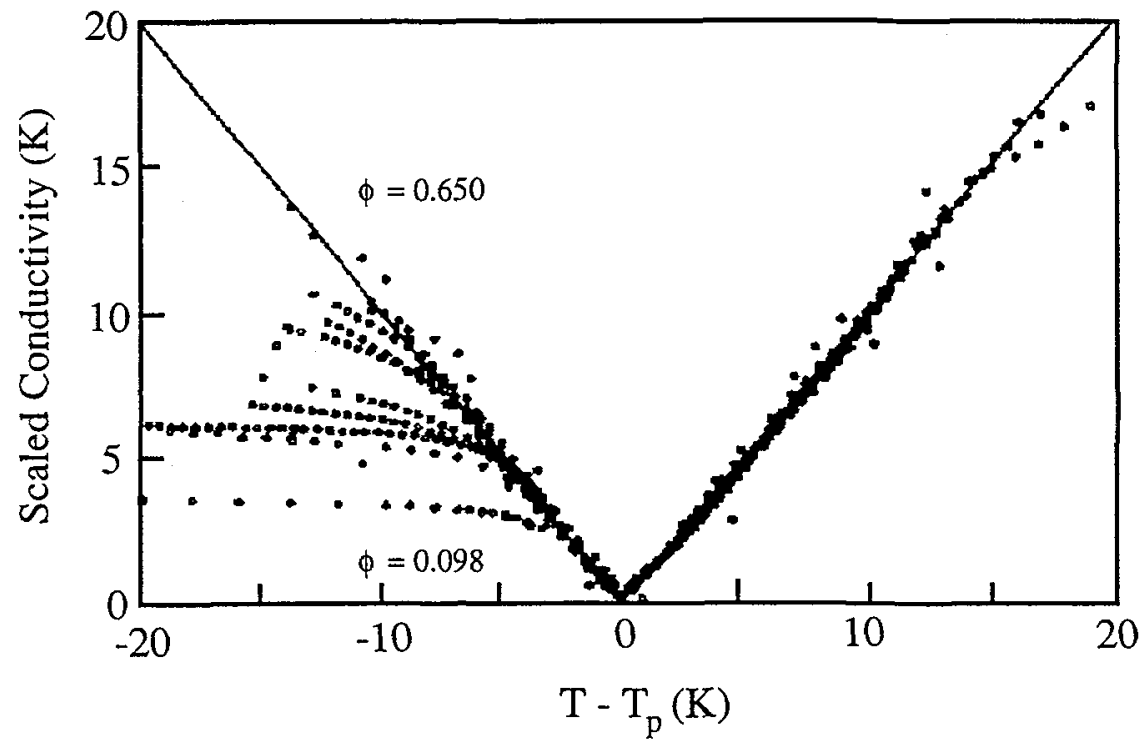

Fig. 3, The scaled conductivity $T_{p}(\sigma / A)^{1 / s^{\prime}}$ and $T_{p}(\sigma / B)^{-1 / t}$ are plotted as a function of $T-T_{p}$ at different volume fractions, showing the power law behavior near $T_{p}$.

We have thus a well-established experimental evidence that the percolation in AOT/water/decane system in the $\mathrm{L}_{2}$ phase is associated with a clustering phenomenon. 
The phase diagram that we depict in Fig.1 should therefore be obtainable from the standard liquid theory with a reasonable definition of the percolation. We shall outline one such theory in the next section.

\section{The Phase Diagram According to Baxter's Sticky Sphere Model}

A reasonable model for a microemulsion in $\mathrm{L}_{2}$ phase is to regard it as a collection of spherical colloidal particles of an average radius $\langle R>$ interacting among one another via a short-range attractive pair-potential. This pair-potential can, for example, be a square-well potential with a hard-core diameter of a, plus an attractive tail of depth $-\varepsilon$ and a width $\Delta$. The liquid theory with a square-well potential in general can not be solved in an analytical form except for a limiting case in which $\varepsilon$ tends to infinity and $\Delta$ to zero in such a way that the contribution to the second virial coefficient exists. This limiting potential is called Baxter's sticky sphere potential. Specifically, the pair-potential is of the form:

$$
\begin{aligned}
\beta \mathbf{u}(\mathbf{r}) & =+ \text { infinity } & & \mathrm{r}<\sigma \\
& =-\operatorname{Ln}[(1 / 12 \tau)(\mathrm{a} /(\mathrm{a}-\sigma))] & & \sigma<\mathrm{r}<\mathrm{a} \\
& =0 & & \mathrm{r}>\mathrm{a}
\end{aligned}
$$

where $\beta=1 / k_{B} T$, a the outer diameter and $\sigma$ the inner diameter of the attractive well. It is understood that the limit $\sigma->a$ is to be taken in the calculation. From our discussion above, it is obvious that $a=2<R>$. The single dimensionless parameter $\tau$ is called the stickiness parameter. The sphere is stickier the smaller $\tau$ is. In the limit $\tau$ tends to infinity, the pair potential reduces to a hard sphere potential. By equating the respective second virial coefficients, one can map the square-well potential into an equivalent sticky sphere potential in the following way:

$$
1 / \tau=12(\Delta / a)(\exp (\beta \varepsilon)-1)
$$

We assume here $a>>\Delta$. It is seen that $1 / \tau$ is larger the larger $\Delta$ or $\varepsilon$ is.

Baxter showed that [12] the Ornstein-Zernike equation using this sticky pairpotential can be solved analytically in the Percus-Yevick approximation [13]. The PY approximation in this case amounts to a reasonable ansatz that the direct correlation function $c(r)=0$ outside the range of the potential a. Combining this ansatz with the exact boundary condition for hard spheres that the pair-correlation function $g(r)=0$ inside the hard core $a$, the direct correlation function inside the hard core can be found. Thus one can obtain an analytical form of the three-dimensional Fourier transform of the direct correlation function $c(k)$ as a function of the volume fraction of the spheres $\eta$ and the stickiness parameter $\tau$. Here, $\eta=\rho a^{3} \pi / 6$, and $\rho$ is the number density of the particles. In comparing the theory with experiments for the scattering intensities, we shall identify $\eta$ with $\phi$. However, for comparison of the measured phase diagram with Baxter's theory we shall chose $\eta=1.7 \phi$. This amounts to choosing a distance $D$ between two percolating spheres $19 \%$ larger than the diameter of the average sphere. The reason for this is that in a continuum percolation theory there is no particular requirement for two spheres to be connected only when they are in close contact.

First, the inter-particle structure factor $S(k)$ is calculated from the relation:

$$
S(k)=1 /[1-\rho c(k)]
$$


From the limiting value $S(k->0)=\rho k_{B} T \chi_{T}$ we can get the isothermal compressibility $\chi_{\mathrm{T}}$. By integrating $\chi_{\mathrm{T}}$ with respect to the number density, one obtains the compressibility equation of states:

$$
\frac{\beta p}{\rho}=\frac{1+\eta+\eta^{2}}{(1-\eta)^{3}}-\frac{\lambda \eta(1-\eta)\left(1+\frac{1}{2} \eta\right)-\lambda^{3} \eta^{2}(1-\eta)^{3} / 36}{(1-\eta)^{3}}
$$

where the parameter $\lambda$ is given by the smaller real root of:

$$
\frac{\eta}{12} \lambda^{2}-\left(\tau+\frac{\eta}{1+\eta}\right) \lambda+\frac{1+\eta / 2}{(1-\eta)^{2}}=0
$$

From the equation of states, one finds the existence of gas-liquid phase transition with a critical point occurring at $\eta_{c}=0.1213$ and $\tau_{c}=0.0976$. Again, by integrating the compressibility equation of states, Barboy [14] was able to obtain an analytical chemical potential $\mu$ valid both in the one- and two-phase regions. Having the chemical potential and pressure, one can then obtain the coexistence curve by solving for the coexisting gas and liquid densities at a given $\tau$, which is less than $\tau_{c}$ in the two-phase region. Figure 4 shows the coexistence line obtained this way in a $\tau / \tau_{c}$ vs $\eta / \eta_{c}$ plot. In the same figure the corresponding spinodal line is also plotted in a dash line. The spinodal line is the loci of $\{\tau, \eta\}$ where the isothermal compressibility diverges. It is seen that the coexistence curve is highly skewed toward the low volume fraction side, a feature which is often seen in micellar solutions and microemulsions. This is due to the interaction which is short range and strong and is in sharp contrast to the well-known Van der waal case, which is derived from an interaction that is long range and weak. To assess the degree of asymmetry on the gas and liquid sides, we have worked out the respective asymptotic behaviors:

$$
\begin{array}{ll}
\left(1-\tau / \tau_{c}\right)=0.1584\left(\eta / \eta_{c}-1\right)^{2} & \eta<\eta_{c} \\
\left(1-\tau / \tau_{c}\right)=0.0264\left(\eta / \eta_{c}-1\right)^{2} & \eta>\eta_{c}
\end{array}
$$

These equations show that the PY approximation gives the mean field exponent $\beta=1 / 2$.

One of the nicest feature of Baxter's model is, however, that one can also derive analytically the percolation loci in the $\{\tau, \eta\}$ plane. Coniglio et al [15] introduced a pairconnectedness function $\mathrm{P}(\mathrm{r})$ in 1977 in connection with development of a continuum percolation theory. Given a particle at the origin, $4 \pi r^{2} \rho P(r) d r$ is the number of particles in the spherical shell $(r, r+d r)$ which are connected to this central particle and belong to the same cluster. Coniglio et al showed that $P(r)$ also satisfied an Ornstein-Zernike type equation with a modified direct correlation function $\mathrm{c}^{+}(\mathrm{r})$. By invoking the short range nature of the direct correlation function, namely, $c^{+}(r)=0$, for $r>a$, and the sticky sphere condition:

$$
\mathrm{P}(\mathrm{r})=(1 / 12) \lambda \mathrm{a} \delta(\mathrm{r}-\mathrm{a})
$$

Chiew and Glandt [16] was able to show that the average cluster size $S$ is given by

$$
S=1 /(1-\lambda \eta)^{2}
$$

The onset of percolation can be defined as the point where $S$ diverges. Thus percolation loci in the $\{\tau, \eta\}$ plane is given by $\eta=1 / \lambda$, leading to an equation: 


$$
\tau=\left(19 \eta^{2}-2 \eta+1\right) / 12(1-\eta)^{2}
$$

Figure 4 shows also a percolation line according to eq.(12).

In order to compare the theoretical phase diagram with the actual one, we have to specify the relationship between the stickiness parameter $\tau$ and the temperature. Eq.(4)

(a)

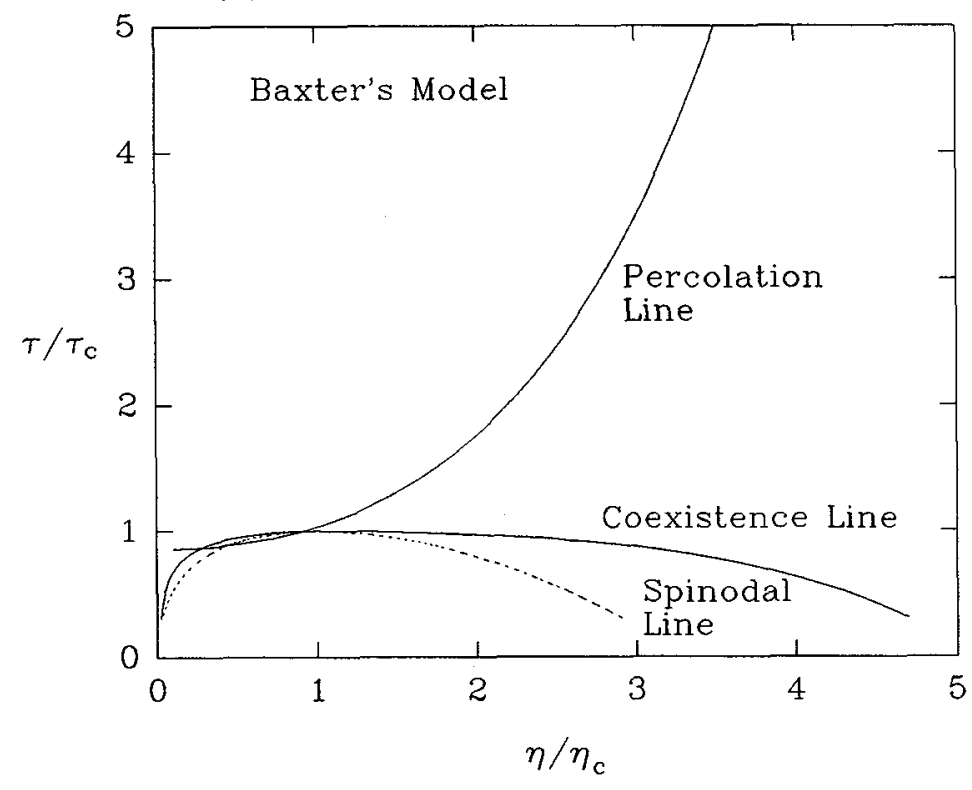

(b)

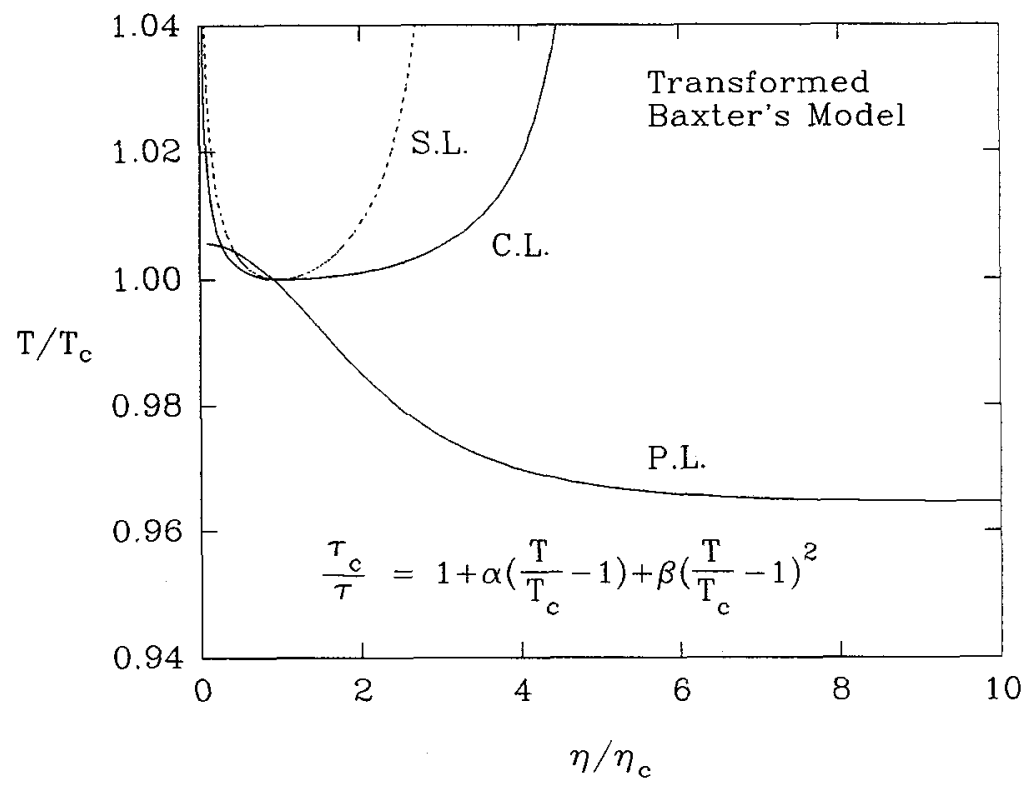

Fig. 4, Theoretical percolation, coexistence and spinodal lines according to Baxter's model. 4 a gives universal relations between $\tau / \tau_{c}$ and $\eta / \eta_{c}$ according to the model. $4 \mathrm{~b}$ shows the results after a transformation given by eq.(13). 
suggest that $1 / \tau$ is proportional to the interaction strength and the interaction strength should increase with temperature. because we have a lower consolute point. The simplest relationship with two parameters $\alpha$ and $\beta$ is

$$
\tau_{c} / \tau=1+\alpha\left(T / T_{C}-1\right)+\beta\left(T / T_{C}-1\right)^{2}
$$

We can try to fit the experimental coexistence curve using eq (13) and then predict the percolation loci with it. Figure 5 shows the results of choosing $\alpha=40$ and $\beta=300$ and $\eta=1.7 \phi$.

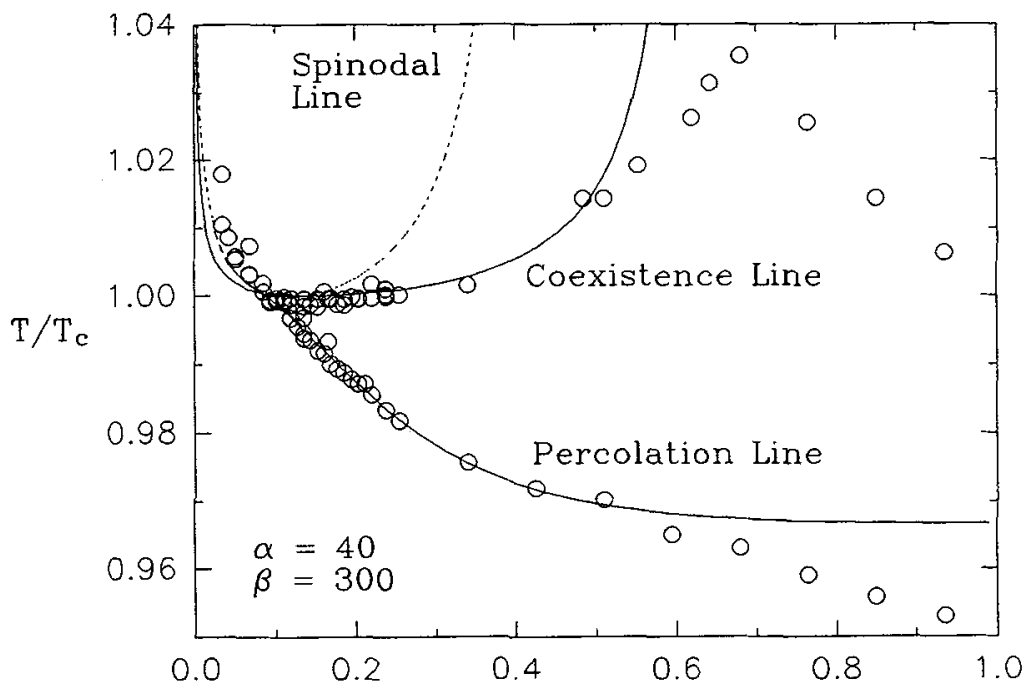

$\eta$

Fig. 5, Experimental data and the corresponding theoretical lines based on Baxter's model after the transformation $\tau_{c} / \tau=1+\alpha\left(T / T_{C}-1\right)+\beta\left(T / T_{C}-1\right)^{2}$, with $\alpha=40$ and $\beta=300$. Experimental $\phi$ have been multiplied by 1.7 to obtain the theoretical $\eta\left(=\pi \rho \mathrm{a}^{3} / 6\right)$ for a better match with theoretical lines.

\section{Analysis of SANS Data Below Tc}

SANS intensity distribution from a system of polydispersed spherical droplets can be written as [17]:

$$
\mathrm{I}(\mathrm{Q})=(\Delta \rho)^{2} \phi_{\mathrm{w}}\left(\frac{4 \pi}{3} \overline{\mathrm{R}}^{3}\right) \frac{(\mathrm{Z}+6)(\mathrm{Z}+5)(\mathrm{Z}+4)}{(\mathrm{Z}+1)^{3}}<\overline{\mathrm{P}}(\mathrm{Q})>\langle\mathrm{S}(\mathrm{Q})>
$$

where $\Delta p=\rho_{w}-\rho_{S}$ is the difference of scattering length densities of $D_{2} O$ and protonated decane, $\phi_{w}$ the volume fraction of $\mathrm{D}_{2} \mathrm{O}, \overline{\mathrm{R}}=\langle\mathrm{R}\rangle$, the average radius of the water core, and $\mathrm{Z}$ the index related to the polydispersity. The normalized, volume square averaged particle structure factor is defined as:

$$
<\overline{\mathrm{P}}(\mathrm{Q})>=<\mathrm{R}^{6}\left[3 \mathrm{j}_{1}(\mathrm{QR}) /(\mathrm{QR})\right]^{2}>/<\mathrm{R}^{6}>
$$

The form factor of a spherical particle of radius $R$ is $F(Q)=3 j_{1}(Q R) /(Q R)$. The form factor averaged inter-particle structure factor is defined as: 


$$
<S(Q)>=\sum_{i, j}^{p}\left(\rho_{i} \rho_{j}\right)^{1 / 2} F_{i}(Q) F_{j}(Q) S_{i j}(Q) / \sum_{i}^{p} \rho_{i} F_{i}^{2}(Q)
$$

The size average is taken with respect to a Schultz distribution, which is known to be accurate in the case of AOT/water/decane system[1]. In this case the degree of polydispersity is $\Delta R / \angle R>=(1+Z)^{-1 / 2}$. The partial structure factor, $S_{i j}(Q)$, for a multicomponent sticky sphere system has been given by Robertus et al [18], for $i, j=1$ to 9 , using Baxter's method. The Fortran package for calculating the partial structure factors has been kindly supplied to us by Dr. J.G.H. Joosten. The volume square averaged particle structure factor, assuming the Schultz distribution of sizes, had previously been given in an analytical form by Kotlarchyk et al [19].

Equation 14 is a three-parameter theory containing three adjustable parameters $<R>$, $\mathrm{Z}$ and $\tau$. These parameters are functions of temperature and volume fraction. Here we assume that particles of different sizes have the same degree of stickiness.

SANS measurements were made at the Low Angle Biology Diffractometer at the High Flux Beam Reactor of Brookhaven National Laboratory. Wave length of neutrons was selected by a multilayer monochrometer at $\lambda=5.35 \mathrm{~A}$ with $\Delta \lambda / \lambda=10 \%$. The sample to detector distance was set at $130 \mathrm{~cm}$ and the $50 \mathrm{~cm}$ by $50 \mathrm{~cm}$ area detector was offset $10 \mathrm{~cm}$ from the center so that the $Q$ range covered from $0.012 \mathrm{~A}^{-1}$ to $0.35 \mathrm{~A}^{-1}$ in a single measurement.

Fig. 6 shows the result of fitting Eq. 14 to the data at $\phi=0.08$ and $T=36.5 \mathrm{C}$. The upper left hand corner shows the $I(Q)$ vs $Q$ plot in a linear scale. Circles are SANS data in absolute scale $\left(\mathrm{cm}^{-1}\right)$ and solid line is the theory. The upper right hand corner shows an
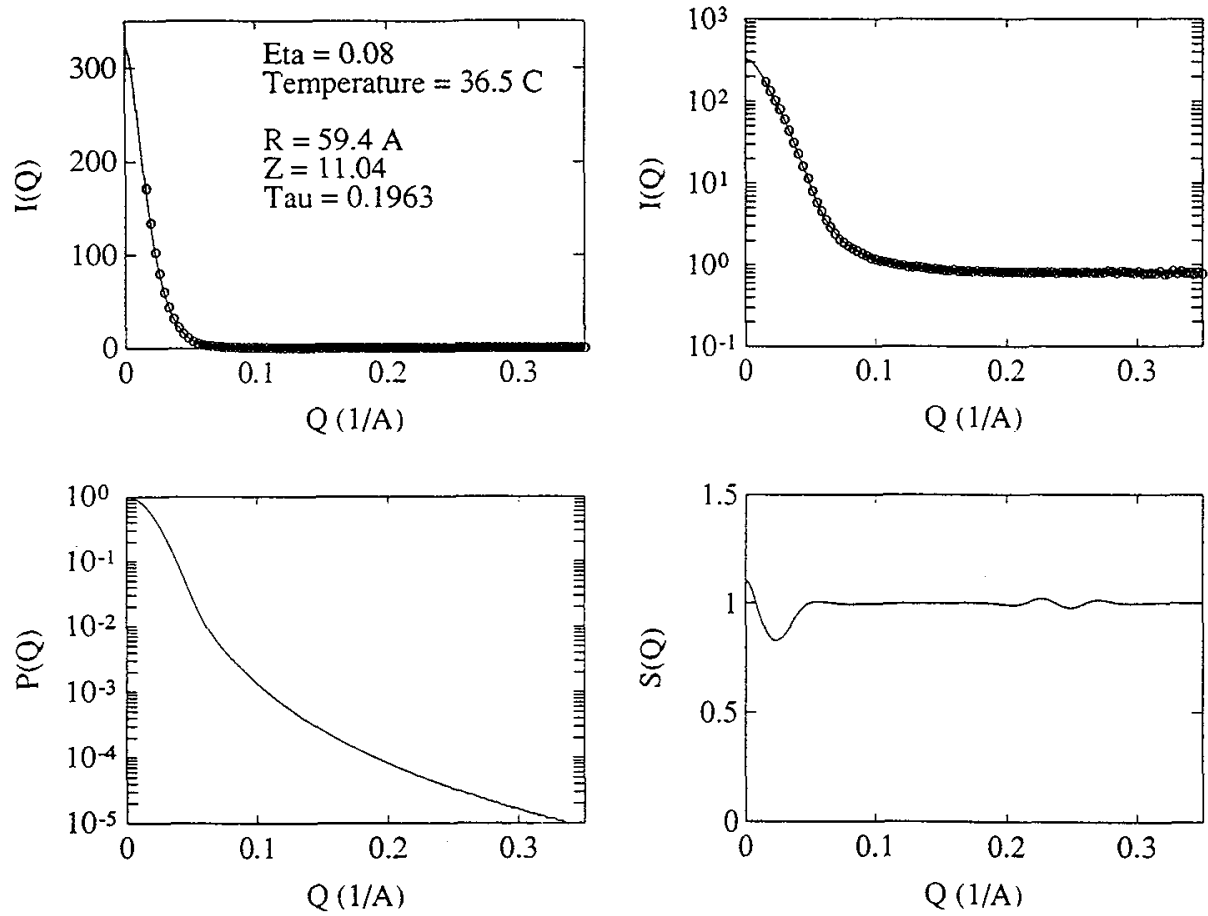

Fig. 6, SANS intensity $\mathrm{I}(\mathrm{Q})$, at $\eta=0.08, T=36.5 \mathrm{C}$, in an absolute scale $\mathrm{cm}^{-1}$ plotted in linear and log scales. Circles are experimental data and the solid line is the fitted curve. Showing below are the particle structure factor (lower left) and the inter-particle structure factor (lower right) extracted from the fitting. 
equivalent plot in a semilog scale. The lower left hand corner depicts the normalized, volume square averaged particle structure factor and the lower right hand corner gives the form factor averaged inter-particle structure factor. The fit is very good and from which we were able to extract $\langle R\rangle=59.4 \mathrm{~A}$, closed to what we estimated in the introduction, and $Z=11.04$, corresponding to a polydispersity index of $28.8 \%$. The dimensionless interaction parameter $\tau=0.1963$, close to the critical value $\tau_{c}$, since the state is very near the critical point. As can be seen, the average inter-particle structure factor shows a zero angle peak due to the critical scattering and is devoid of the first diffraction peak due to the low volume fraction. The slight wiggle around $Q=0.25$ is an artifact due to a finite sampling ( $\mathrm{p}=9$ ) of the Schultz size distribution function. It does not affect the quality of the fit because the average particle structure factor already decays to below $10^{-4}$ in this $Q$ range.

Fig. 7 shows the fits to the other four lower temperature cases at the same volume fraction and their results. Upper left-hand corner displays the semilog plots of the intensities, each curve above $20 \mathrm{C}$ being displaced upward by a factor five for the display purpose. Upper right-hand corner depicts variation of $\langle R>$ as a function of temperature. Lower right-hand corner the same thing for $Z$. It is worth noting that as temperature increases the average water droplet size decreases and the size distribution slightly broadens, an expected trend in a micellar system. What is most pleasing to see is, however, that the temperature variation of $\tau_{c} / \tau$ comes out in the same form as eq. 13 except close to the critical point. This is shown in lower left-hand corner. Circles are results of the fits and the solid line is eq. 13 with $\alpha=21.4$ and $\beta=133.3$. These coefficients are different in values from those obtained from fitting the theory to the cloud point curve above $T_{\mathcal{c}}$. This situation is similar to the case of non-ionic micellar solution investigated by Monohar et al [20].
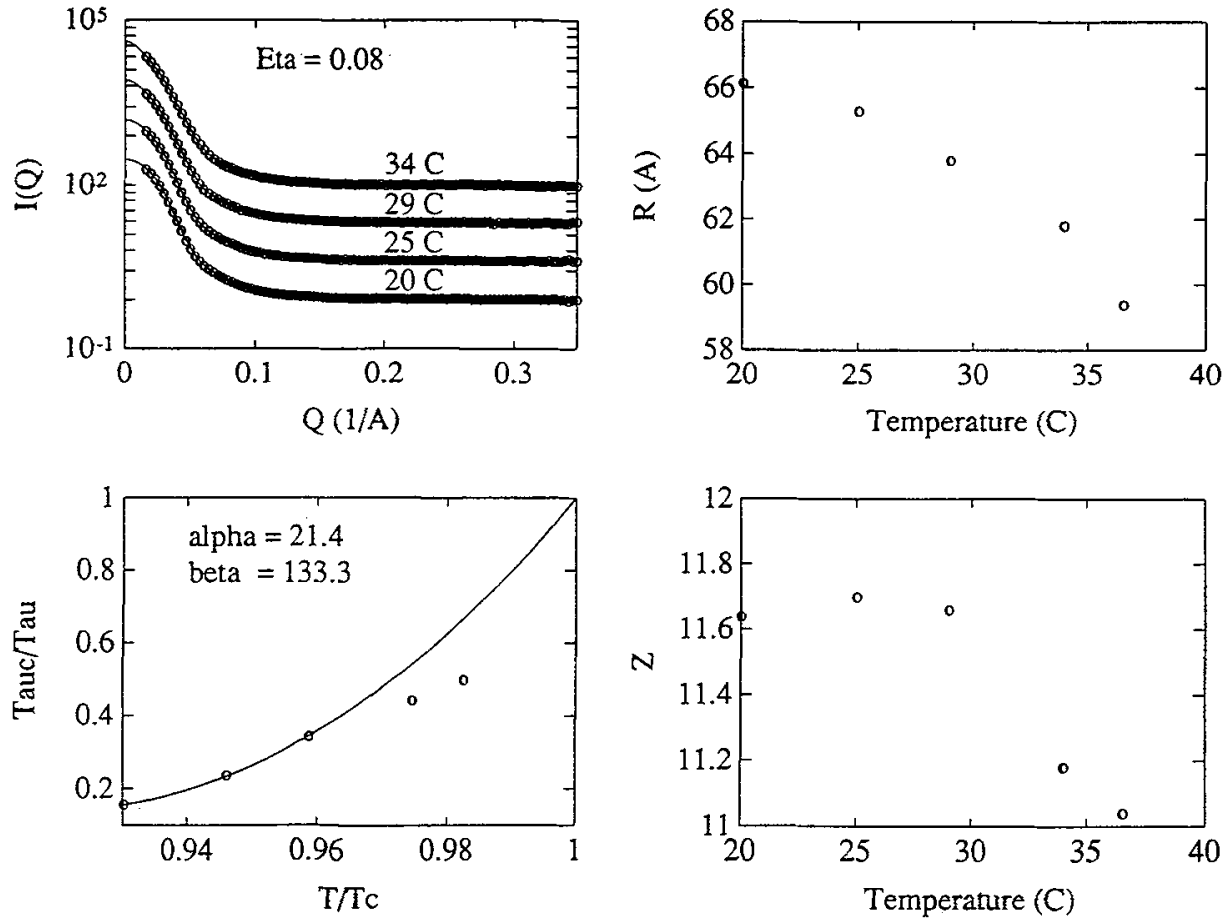

Fig. 7, SANS data analysis of samples with $\eta=0.08$. Upper left: the results of the fits to intensities at different temperatures. Upper right: $\left\langle R>\right.$ vs Temperature. Lower left: $\tau_{c} / \tau$ vs $T / T_{C}$. Open circles are the relative stickiness parameters obtained from the fittings of data at different temperature. The solid line is the prediction of eq.(13) with $\alpha=21.4, \beta=133.3$. Lower right: $Z$ vs Temperature. 
We next focus our attention to a typical high volume fraction region where the Percus-Yevick approximation for hard-sphere system is sill accurate. Specifically, we discuss the case of $\phi=0.50$. Recall from Fig. 1, the percolation temperature is about $28 \mathrm{C}$ at this volume fraction. Fig. 8 shows a sequence of similar plots as in Fig. 6, but for $\phi=0.5$. Note the difference between the high and the low volume fraction cases: the inter-particle structure factor shows a first diffraction peak rather than a zero angle peak, because we are at a high volume fraction and far away from the critical point. Note also that the average structure factor has only the first diffraction peak and all the subsequent higher order peaks are washed out by the polydispersity. The average water core radius is $71.6 \mathrm{~A}$ and the polydispersity index $28.4 \%$. Fig. 9 shows the results of the analyses for all the temperatures at $\phi=0.50$. The upper left-hand corner displays the absolute intensities in a semilog scale. Each curve above $25 \mathrm{C}$ is displaced upward by a factor ten for displaying purpose. The average water core radius decreases and the size distribution broadens as the temperature is raised, similar to the low volume fraction case. However, the stickiness parameter $\tau$ stays essentially constant at 100, indicating that the system behaves like hard spheres at high volume fractions, independent of temperature.
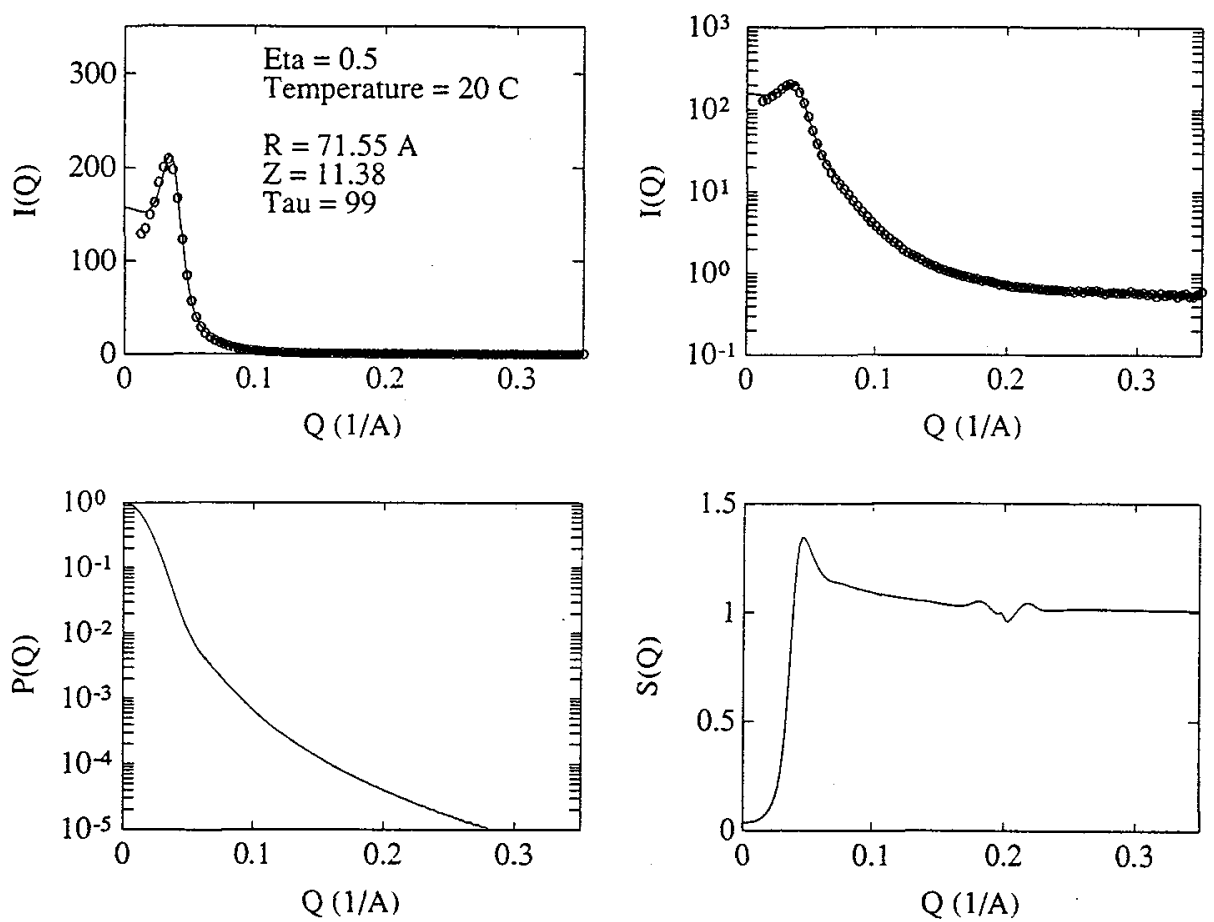

Fig. 8, SANS intensity $\mathrm{I}(Q)$ at $\eta=0.5, T=20 \mathrm{C}$, in an absolute scale $\mathrm{cm}^{-1}$ plotted in linear and log scales. Circles are experimental data and the solid line is the fitted curve. Showing below are the particle structure factor (lower left) and the inter-particle structure factor (lower right) extracted from the fit.

In order to assess the adequacy of the method of data analyses using the polydispersed sticky sphere model given above, we present in Fig. 10 an alternative method of analysis using a Teubner-Strey model [21]. T-S model is applicable when the microstructure of a microemulsion is bicontinuous [5,6]. The upper half of Fig. 10 displays results of the T-S analysis, in both linear and semilog plots. The bottom half of the figure gives results of the polydispersed hard-sphere model for comparison. Chi squares result from the two methods of fits are 51.5 and 8.2 respectively. This comparison clearly 

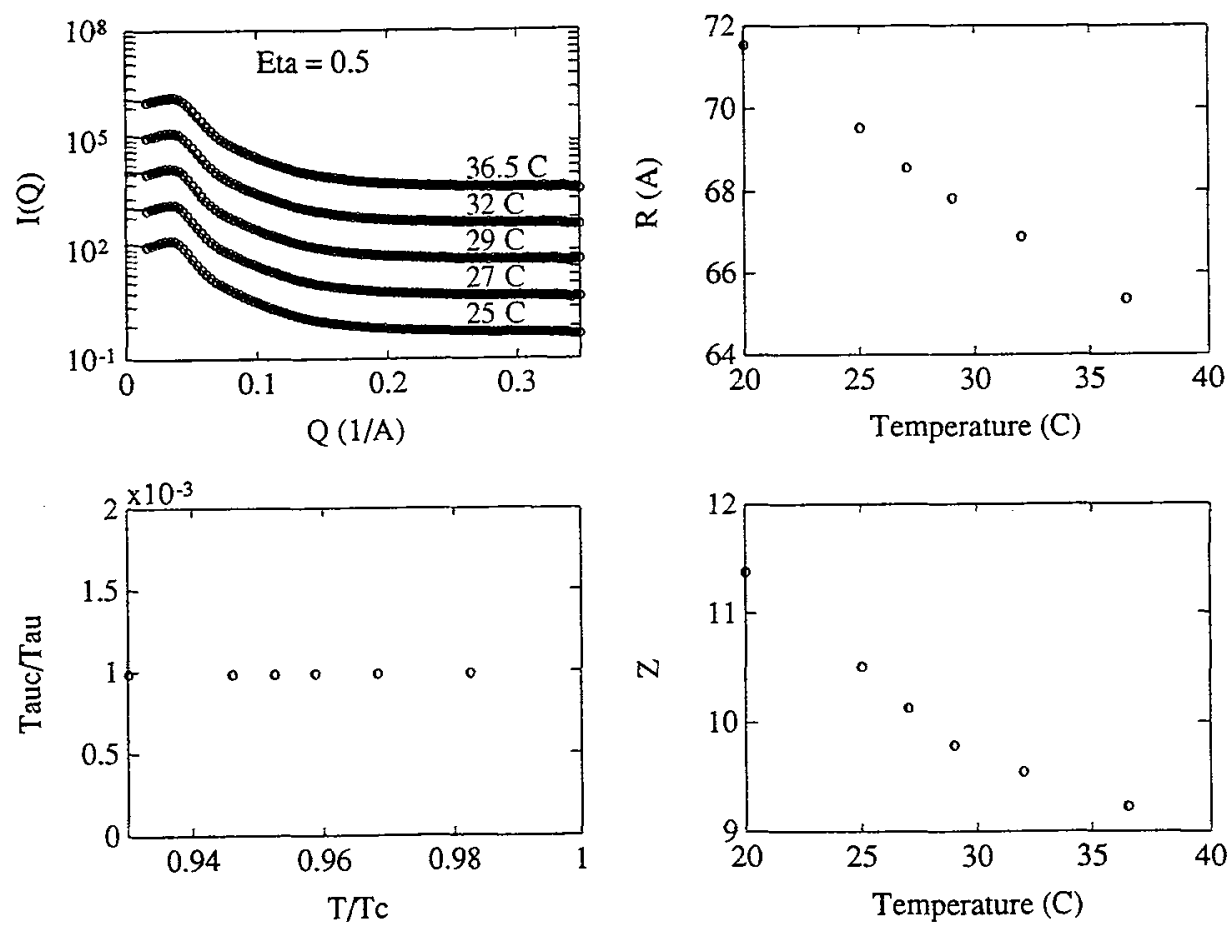

Fig. 9, SANS data analysis of samples with $\eta=0.5$. Upper left: the results of the fits to intensities at different temperatures. Upper right: $\angle R>$ vs Temperature. Lower left: $\tau_{C} / \tau v s T / T_{C}$. Open circles are the relative stickiness parameters obtained from the fittings of data at different temperature. Lower right: $\mathbf{Z}$ vs Temperature.

indicates that for a microemulsion at $\phi=0.50$ and $T=20 \mathrm{C}$, the polydispersed hard-sphere model is more adequate.

On the other hand, when the temperature is much above the percolation threshold, the applicability of the T-S model can be shown to become evident. Fig. 11 gives a comparison of the analyses using the two models for the case of $\phi=0.5$ and $T=36.5 \mathrm{C}$. The upper half of the figure shows the T-S model analysis and the bottom half the analysis using the polydispersed hard-sphere model. The chi squares are 35.8 and 21.5 respectively. We judge that the two model fits are equally satisfactory. In the T-S model, the two parameters extracted are: $k=2 \pi / d$ and $\xi$ [21]. We previously gave a physical interpretation of the two parameters $d$ and $\xi$ for the case of microemulsions with equal volume fractions of water and oil. $\mathrm{d}$ is the average inter-domain distance between two adjacent water (or oil) domains in a bicontinuous structure. Although the parameter $\xi$ has no direct physical meaning, the dimensionless parameter $1 / \mathrm{k} \xi$ is proportional to the polydispersity of the domain sizes [22]. For the cases we discuss here, we can take $k \xi$ as an order parameter representing the degree of order in the bicontinuous structure. For example as we approach the lamellar boundary, this order parameter should increase. This is indeed the case for $\phi=0.50$ microemulsions. As we go above the percolation temperature $28 \mathrm{C}$, where the T-S model becomes applicable, magnitude of the order parameter is seen to increase as the temperature goes higher. 

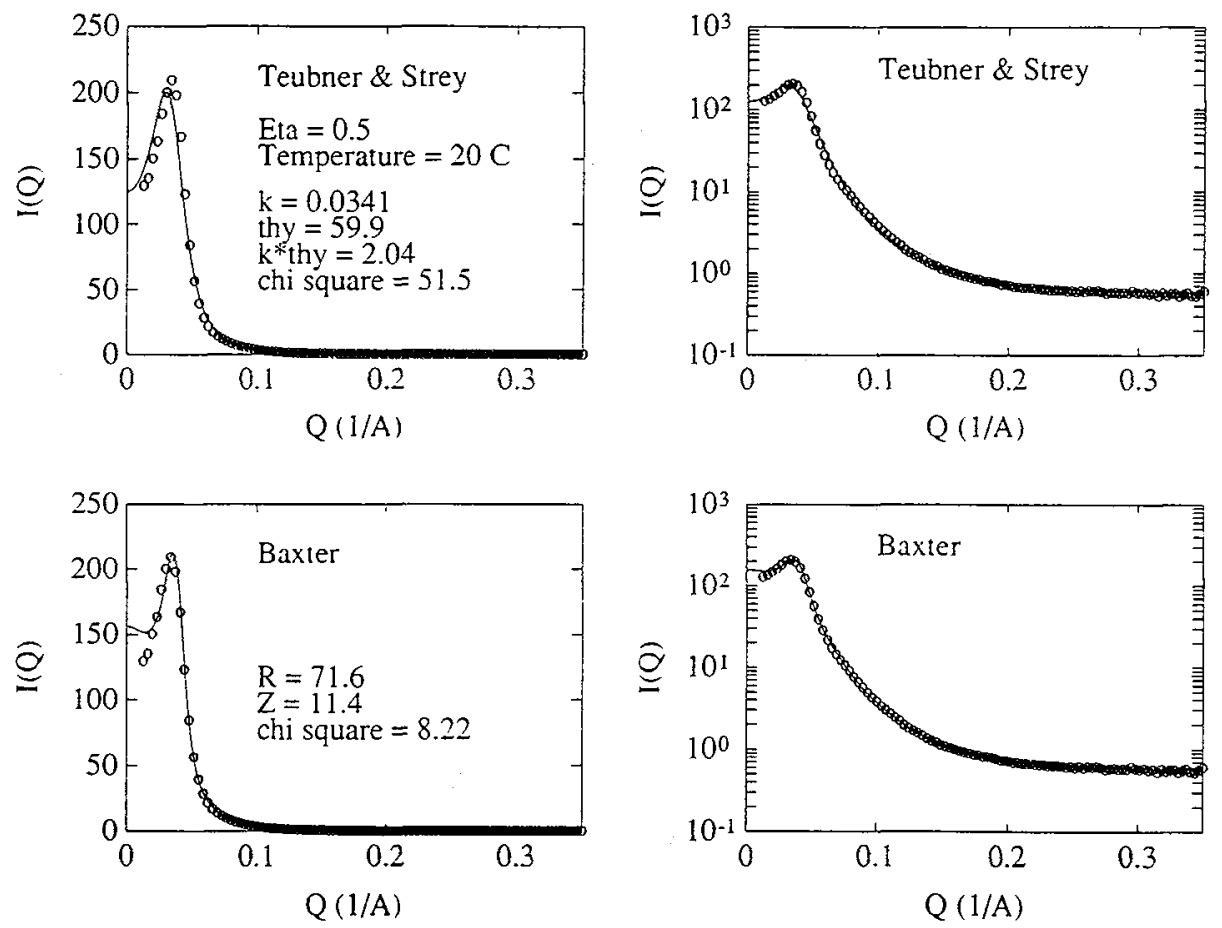

Fig.10, Comparison of results of fittings from two different models with the same set of data at $\eta=0.5$ at $20 \mathrm{C}$. Upper part is the result of fitting with Teubner-Strey's model. Lower part is the result of fitting with Baxter's Model. This shows the Baxter's model is more appropriate for the lower temperatures.

\section{Dynamics of the Droplet Number Density Fluctuation}

We shall turn next to discussion of some aspects of the droplet dynamics near the percolation threshold. In 1985, Chen and Huang [23] observed an interesting dynamic slowing-down phenomenon in the $\mathrm{AOT} / \mathrm{H}_{2} \mathrm{O} /$ decane system at $\mathrm{W}=40$, using photon correlation spectroscopy. Maintaining the temperature at $23 \mathrm{C}$, they measured a series of droplet density time correlation functions as a function of the droplet volume fraction. As $\phi$ was increased from 0.10 to 0.75 , the first cumulant of the time correlation function continuously decreased until $\phi=0.65$ and then increased slightly after that. The cause of the slowing-down of the relaxation rate was at first attributed to the structural arrest in dense liquids due to the cage effect [24]. However, some experimental observations were inconsistent with the details of the predictions of the so called glass transition theory [25] in dense or supercooled liquids. Firstly, the microemulsion at $\phi=0.65$ is still a fluid, although the viscosity of it is about 100 fold greater than that of the pure decane. For a hard sphere system it is known [25] that the dynamic glass transition occurs at a packing fraction of about 0.5 . So at 0.65 the system would have been a solid with a viscosity much more than 100 Poise. Secondly, the first cumulant showed an upturn after the volume fraction of 0.65 , which is inconsistent with the notion that the structural arrest has occurred at $\phi=0.65$. Thirdly, the time correlation functions clearly showed an initial exponential decay at short time, with a well defined first cumulant, and then gradually evolved into a stretch exponential at longer time with a stretch exponent $\beta$ varies between 0.9 and 0.6 , depending on the volume fraction. Most puzzling fact was that the exponent $\beta$ seemed to vary with the scattering angle, namely $Q$ [17], which is against the intuition that the exponent ought to be universal. The existence of the percolation line in this system was not realized until 

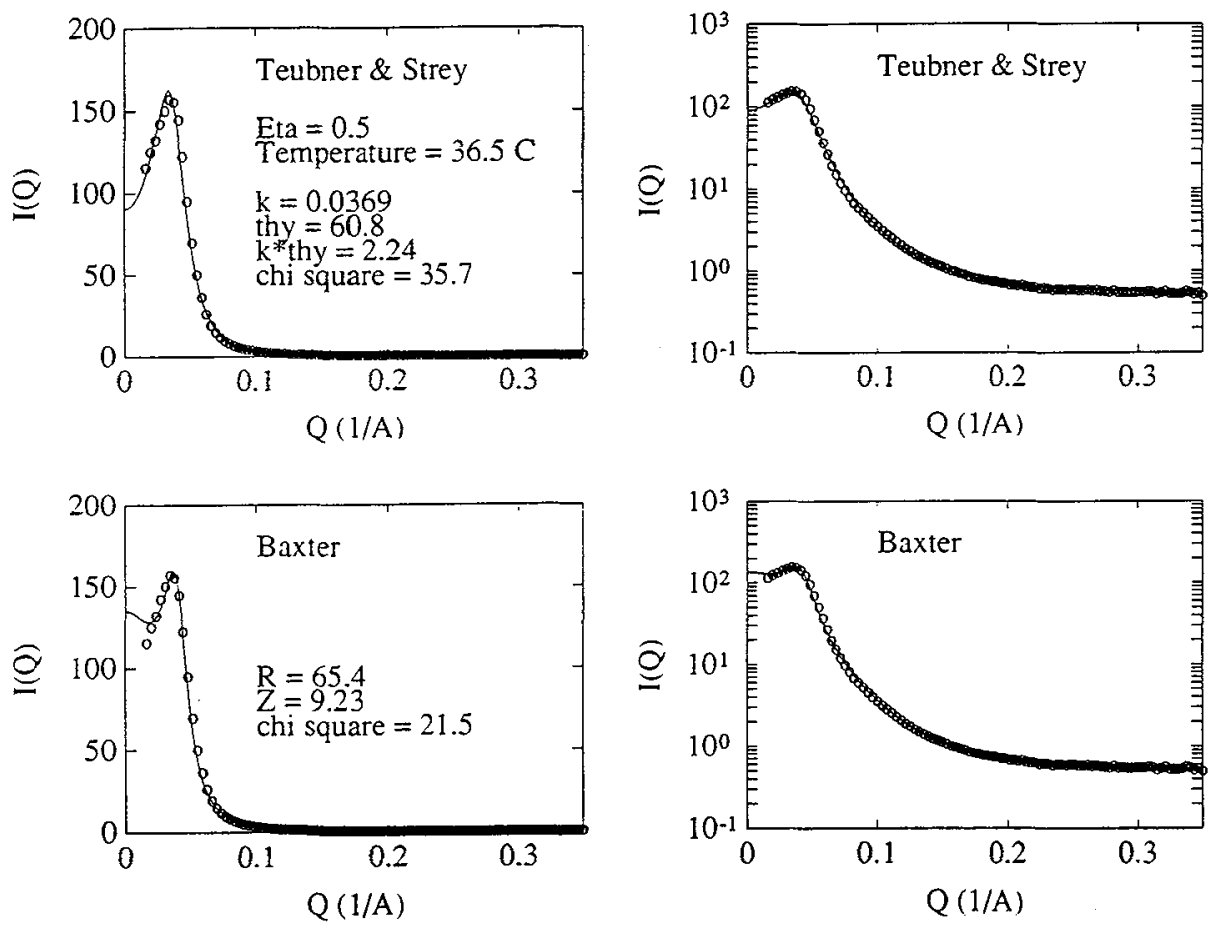

Fig.11, Comparison of results of fittings from two different models with the same set of data at $\eta=0.5$ at $36.5 \mathrm{C}$. Upper part is the result of fitting with Teubner-Strey's model. Lower part is the result of fitting with Baxter's Model. This shows the two models are equally appropriate at higher temperatures.

about 1990 [8]. From Fig. 1, it can be seen that at $23 \mathrm{C}$ the percolation threshold occurs at a volume fraction of about 0.65 . This may be the cause of the dynamic slowing-down. We shall outline below a theory of dynamic slowing-down, based on the percolation phenomenon, which is capable of explaining all the observed facts of the photon correlation measurements [26].

The starting point of our theory is an assumption that the dynamics of the droplets is dominated by diffusional motion of the percolation clusters. This assumption is expected to be good in the vicinity of the percolation threshold where large, transient fractal clusters are formed. Formation of the transient fractal clusters is a necessary condition for the dynamic percolation theory [9] to be valid, and which we have used to explain the conductivity exponent below the threshold in the introduction.

In light scattering, the wave length of light is much greater than the droplet size. Hence the particle structure factor is near unity and we can ignore it. First, we calculate the inter-particle structure factor $S_{k}(Q)$ for a cluster containing $k$ particles. We do this by Fourier transforming the cluster pair correlation function [27]:

$$
\rho g_{k}(r)=\frac{D}{4 \pi R_{1}^{D}} \frac{\exp \left(-r / R_{k}\right)}{r^{3-D}}
$$

where $D$ is the fractal dimension of the clusters, $R_{k}=R_{1} k^{1 / D}$, the dimension of the $k$ cluster, and $R_{1}$ the average radius of the droplet. The result can be put in an analytical form 


$$
\mathrm{S}_{\mathrm{k}}(\mathrm{Q})=\frac{\mathrm{k} \sin \left[(\mathrm{D}-1) \arctan \left(\mathrm{QR}_{\mathrm{k}}\right)\right]}{(\mathrm{D}-1) \mathrm{QR}_{\mathrm{k}}\left(1+\mathrm{Q}^{2} \mathrm{R}_{\mathrm{k}}{ }^{2}\right)^{(\mathrm{D}-1) / 2}}
$$

With the structure factor, the intermediate scattering function $I(Q, t)$ can be written as

$$
I(Q, t)=\sum_{\mathbf{k}} \mathrm{kS}_{\mathrm{k}}(\mathrm{Q}) \exp \left(-\mathrm{D}_{\mathrm{k}} \mathrm{Q}^{2} \mathrm{t}\right)
$$

The discrete sum can be converted into an integral over $\mathrm{k}$ by introducing a cluster size distribution function of the form: $N(k)=k^{-\tau} \exp (-k / S)$ [28]. In this expression $\tau$ is the polydispersity exponent, $S$ the average cluster size and $D_{k}=D_{1} k^{-1 / D}$ the translational diffusion coefficient of the k-cluster. Numerical simulations for three-dimensional percolation clusters gave a fractal dimension $\mathrm{D}=2.5$ and the polydispersity exponent $\tau=2.2$ [29]. The measured photon correlation function is then given by $C(Q, t)=I(Q, t) / I(Q, 0)$.

The first cumulant, or the average relaxation rate, is the logarithmic derivative of $C$ $(Q, t)$ evaluated at $t=0$. It is given by:

$$
\Gamma(\mathrm{Q})=\frac{1}{\mathrm{I}(\mathrm{Q}, 0)} \int_{1}^{\infty} \mathrm{dkN}(\mathrm{k}) \mathrm{k}^{2} \mathrm{~S}_{\mathrm{k}}(\mathrm{Q}) \mathrm{D}_{\mathrm{k}} \mathrm{Q}^{2}
$$

\section{A. Dynamic Slowing-Down of the Relaxation Rate}

$\mathrm{Eq}(20)$ for the average relaxation rate $\Gamma(Q)$ can be expressed analytically in a scale variable $x=Q \xi$, where the correlation length $\xi=R_{1}[D(D+1)]^{1 / 2} S^{1 / D}$. The complete analytical form of the relaxation rate $\Gamma(Q)$ has been given in ref. 26 . Here we shall quote only the expressions for the two asymptotic limits. The universal part of the scaling function can be defined as: $\Gamma^{*}(x)=\Gamma(Q) / D_{1} R_{1} Q^{3}$. Then

$$
\begin{array}{ll}
\text { For } x<<1 & \Gamma^{*}(x)=a / x \\
\text { For } x>>1 & \Gamma^{*}(x)=b
\end{array}
$$

where $\mathrm{a}$ and $\mathrm{b}$ are known constants. Fig.12a illustrates the crossover from the small $\mathrm{x}$ behavior of eq. (21) to the large $x$ behavior of eq. (22) using data taken at high volume fractions. Fig. $12 \mathrm{~b}$ gives, as a comparison, the well-known critical slowing-down of therelaxation rate as we approach the critical point at $\phi_{C}=0.10, T_{C}=40 \mathrm{C}$ from below. These data were taken from light scattering work of Rouch et al [30].

\section{B. Stretched Exponential Decay of the Time Correlation Function at Long Time}

Again Eq. (19) in its integral form can be computed analytically [26]. We then obtain the time correlation function in terms of two scaling variables $x$ and $v=D_{1} Q^{2}+S^{-1 / D}=$ $D_{1} R_{1}[D(D+1) / 6]^{1 / 2} Q^{2} t / \xi$. It is sufficient for the purpose here to mention that at sufficiently long time and large $x$, the time correlation function approaches an asymptotic form:

$$
C(x, v)=\exp \left[-(D+1)(v / D)^{\beta}\right]
$$

where the exponent $\beta=D /(D+1)$ is a universal number 0.713 .

The cross-over from the short-time exponential decay $\exp (-\Gamma \mathrm{t})$ to the long-time stretched exponential decay of eq. (23) can be nicely illustrated by plotting a double logarithm of the inverse time correlation function versus logarithm of a dimensionless 
variable $\Gamma t=\Gamma^{*}\left\{x v /[D(D+1) / 6]^{1 / 2}\right\}$. Since $\Gamma^{*}$ is a universal function of $x$ and $D$ is a constant, $\Gamma t$ is a function of $v$ for a given $x$. Thus both asymptotic forms, at short time and at long time, of the droplet density time correlation function can be accommodated by plotting it as a function of $\Gamma t$, at a given $x$. Fig. 13 shows three such plots for $x=0,10$ and 100 . It is seen that the cross-over occurs at approximately $\Gamma t=1$. The solid lines are the theory and
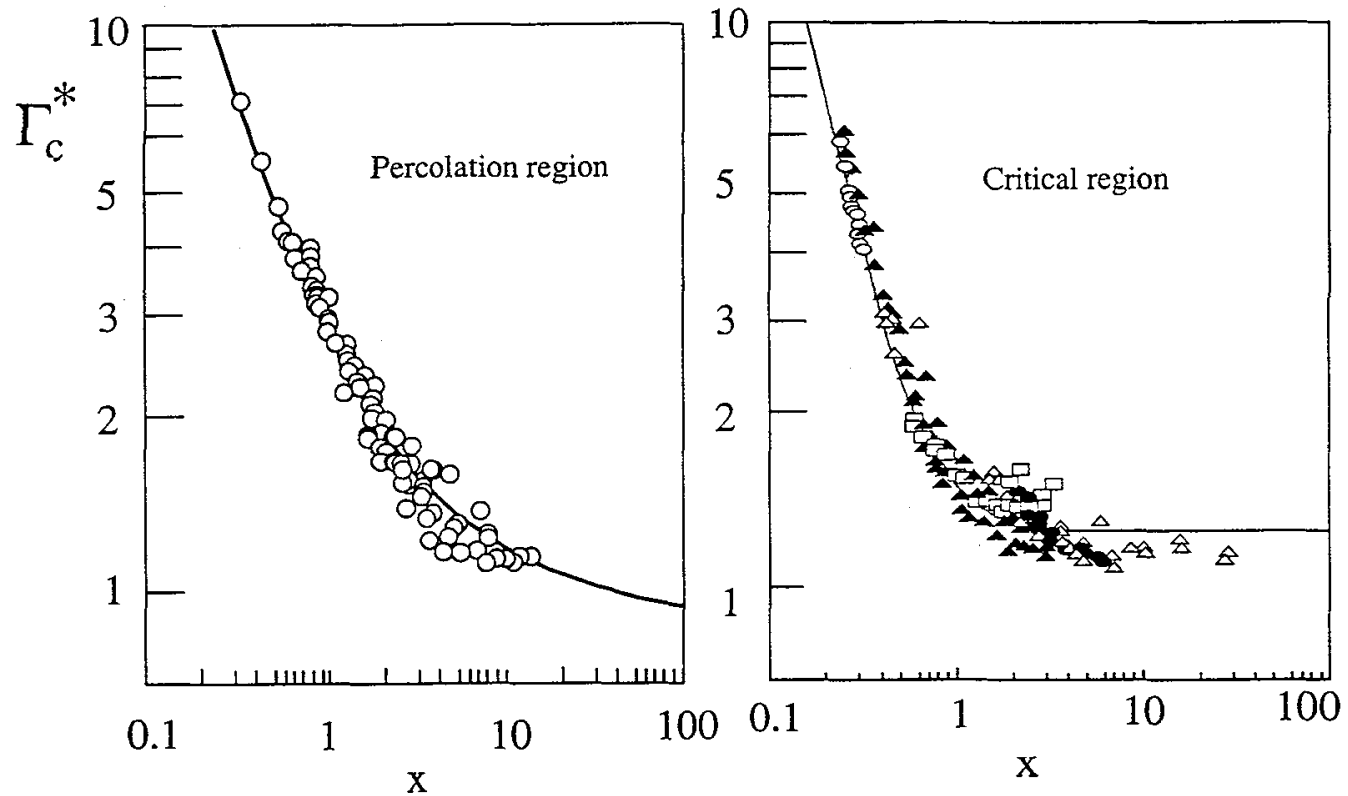

Fig.12, Comparison of dynamic slowing-down in percolation and critical regime. The $\Gamma^{*}(x)$ plotted as a function of the scale variable $x=Q \xi$. Circles are experimental data of reduced relaxation rate at high volume fractions and the solid line is eq.(21), (22). The right hand side figure shows a comparison of the critical slowing-down plot of the low volume fraction data, the solid line is the well-know Kawasaki function.

the circles are the available experimental time correlation functions. It should be noted that in order to cover the two asymptotic regions, experimental photon correlation functions have to be measured over the time range from microseconds to ten seconds. Thus, in practice, it is very difficult to measure a reliable value of the universal exponent $\beta$. In this connection, it is also easy to understand why the earlier measurements of photon correlation functions gave variable $\beta$, depending on both the volume fraction and $Q$. This is due to the fact that the time span of the measured correlation functions covered only microseconds to milliseconds. This situation corresponds to the data indicated in Fig. 13, where all of them fell in the cross-over regime. Thus depending on the value of $\Gamma$ each has, the fitted value of $\beta$ is different. The upturn of the relaxation rate after $\phi=0.65$ at $\mathrm{T}=$ $25 \mathrm{C}$ is due to change of the microstructure of microemulsions from the droplet structure to a bicontinuous structure upon crossing the percolation line.

One last thing we need to mention is the practical way of computing the scale variable $x$. We have to have a method of determining experimentally the correlation length $\xi$ in order to compute $x$. According to a published data of Magazu et al [31], the small angle light scattering intensity showed a definite peak at $Q=0$. The intensity of this peak increases and the width decreases with the approach to the percolation threshold $\phi_{\mathrm{p}}$ at constant temperature. This fact is illustrated by Fig. 14 . At a temperature $T=25 \mathrm{C}$, the small angle light scatterings were measured as a function of $\phi$ from 0.05 to 0.48 [32]. Fig. 14a shows 
the peaking of the intensity on approaching the critical volume fraction $\phi_{\mathrm{C}}$, a regular critical scattering. Fig. 14b, on the other hand, shows the similar peaking on approaching the percolation threshold ( $\phi_{\mathrm{p}}$ around 0.59 ). In analogy with the critical scattering, we can describe the scattering intensity near the percolation threshold by an Ornstein-Zernike equation:

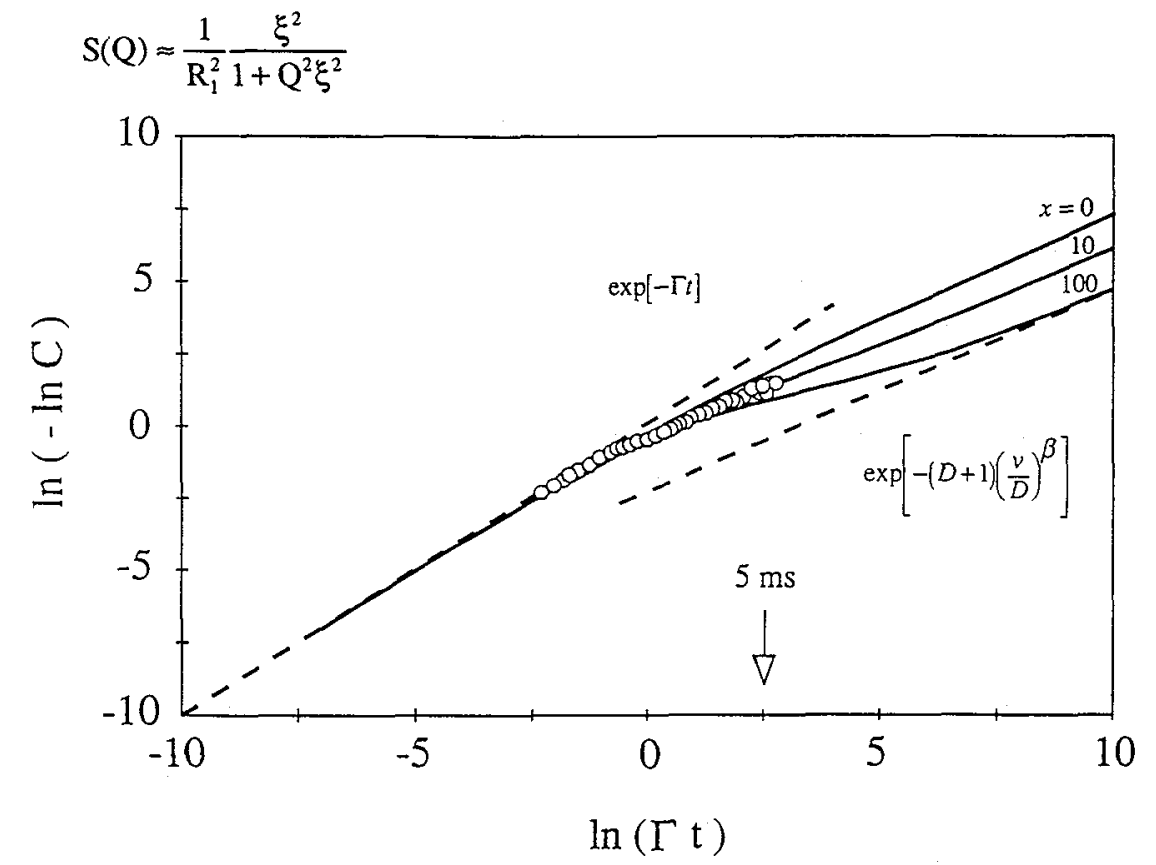

Fig.13, The double $\log$ plot of the inverse universal time correlation function $C(x, v)$ as a function of logarithm of the dimensionless variable $\Gamma t$ for three values of $x=0,10,100$. Note the crossover from exponential to stretched exponential behavior occurring at $\Gamma t=1$. Circles are the available experimental data at $25 \mathrm{C}$.
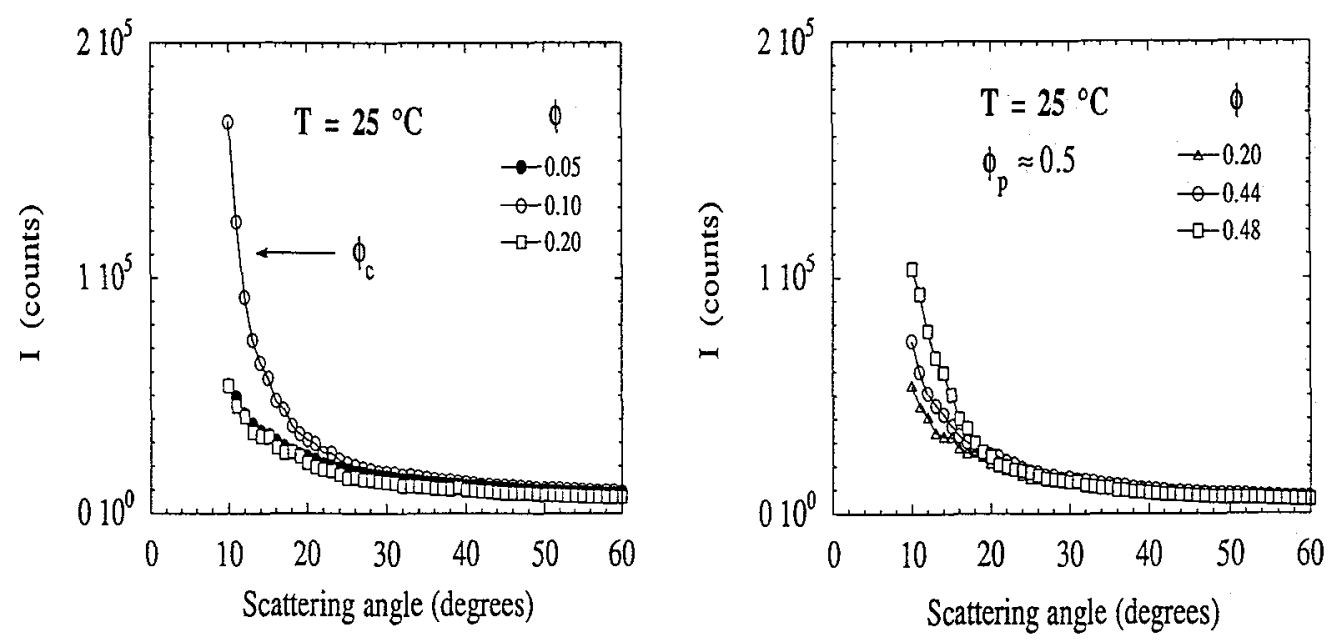

Fig.14, Low angle light scattering intensity vs scattering angle. Note the critical scattering at $\phi_{C}=0.10$ and the excess scattering near the percolation threshold at $\phi_{\mathrm{p}}=0.59$ [32] is visible. 
the particle structure factor being near unity for the light scattering. Using this relation Tartaglia, Rouch and Chen [26] was able to deduce the volume fraction dependence of the correlation length at $25 \mathrm{C}$ to be:

$$
\xi=257(0.59-\phi)^{-0.88} \AA
$$

The correlation length calculated from eq. (25) was used to construct Fig. 12 and 13.

\section{Conclusion}

We have given concrete evidence in this paper that the electrical percolation phenomenon observed in a three-component microemulsion system, AOT/water/decane, can be understood in terms of formation of transient polydispersed fractal clusters due to short-range attraction between microemulsion droplets. This attractive interaction increases in a certain way as the temperature increases. We gave a quantitative relation between the interaction strength and the temperature. This relation serves to explain the over-all feature of the phase diagram, including the cloud-point curve and the percolation line. Analyses of SANS data along a constant low volume fraction line below the critical temperature confirm such a functional relationship. On the other hand, analyses of SANS data a high volume fraction indicate that, away from the critical point, the short-range inter-droplet structure is dominated by the hard-core interaction. However the $30 \%$ or so polydispersity washes out the intermediate-range structure and only the first diffraction peak in the inter-particle structure factor is discernible. The average droplet radius is seen to decrease with increasing temperature. Baxter's sticky sphere model, although successful at low volume fraction near the critical point, cannot reproduce the small angle light scattering peak near the percolation threshold, observed in the experiment of Magazu et al [31]. Without existence of this peak, the correlation length $\xi$, which is an essential ingredient entering the scaling relation governing the dynamic slowing-down of the droplet density relaxation rate, cannot be introduced. It is thus utmost importance that some independent low angle light scattering experiments be done in the near future to further confirm the existence of this low angle intensity peak.

\section{Acknowledgment}

Research of S. H. Chen is supported by a grant from Material Science Division of the U. S. Department of Energy. We are grateful for allocation of neutron beam time at the Low Angle Biology Diffractometer of Brookhaven National Laboratory. We appreciate technical assistance from Dr. D. Schneider during the SANS measurements. 


\section{References}

1. M.Kotlarchyk, S.H.Chen, J.S.Huang and M.W.Kim, Phys.Rev. $\underline{\text { A29, }} 2054$ (1984).

2. M.Kotlarchyk, S.H.Chen, J.S.Huang and M.W.Kim, Phys.Rev.Lett. 53 , 914 (1984).

3. L.Auvray, J.P.Cotton, R.Ober and C.Taupin, J.Phys.Chem. $\underline{88}, 4586$ (1984).

4. F. Lichterfeld, T. Schumeling and R. Strey, J Phys. Chem. 90. 5762 (1986).

5. S.H.Chen, S.L.Chang and R.Strey, J.Chem.Phys. 23,1907 (1990).

6. S.H.Chen, S.L.Chang, R.Strey, J.Samseth and K.Mortensen, J.Phys.Chem. 957427 (1991).

7. J.Rouch, A.Safouane, P.Tartaglia and S.H.Chen, J.Chem.Phys. 90, 3756 (1990).

8. C.Cametti, P.Codastefano, P.Tartaglia J.Rouch and S.H.Chen, Phys.Rev.Lett. 64,1461 (1990).

9. G.S. Grest, I. Webman, S. Safran and A.L.R. Bug, Phys.Rev. A33, 2842 (1986).

10. M.Lagues, J.Phys.(Paris)Lett. 40, L331 (1979).

11. J.P.Clerc, G.Giraud, J.M.Laugier and J.M.Luck, Advances in Phys., 39, 191 (1990).

12. R.J.Baxter, J Chem. Phys. $\underline{49}, 2770$ (1968).

Coniglio, U. De Angelis and A. Forlani, J. Phys. A10, 1123 (1977).

13. J.K.Percus and G.J. Yevick, Phys. Rev. 110, 1 (1958).

14. B.Barboy, J. Chem. Phys. 61 , 3194 (1974).

15. A.Coniglio, U. De Angelis and A. Forlani, J. Phys. A10, 1123 (1977).

16. Y.C.Chiew and E.D.Glandt, J. Phys. A16, 2599 (1983).

17. E.Y. Sheu and S.H. Chen, J. S, Huang and Y. C. Sung, Phys. Rev. A39, 5867 (1989).

18. C. Robertus,W.H. Philipse, J.G.H. Joosten and Y.K. Levine, J. Chem. Phys. 90,4482 (1989).

19. M. Kotlarchyk and S.H. Chen, J. Chem. Phys. 79, 2461(1983).

20. S.V.G. Menon, V. K. Kelker and C. Manohar, Phs. Rev. A43, 1130 (1990).

21. M. Teubner and R. Strey, J. Chem. Phys. 87, 3195 (1987).

22. S. H. Chen, S. L. Chang and R. Strey, Progr. Collid and Polym. Sci. 81,30 (1990).

23. S.H. Chen and J.S. Huang, Phys. Rev. Lett. 55, 1888 (1985).

24.E. Leutheusser, Phys. Rev. A29, 2765 (1984).

25. V. Bengtzelius, W. Goeze and A. Sjolander, J. Phys. C17, 5915 (1984).

26. P. Tartaglia, J. Rouch and S. H. Chen, Phys. Rev. A45, 7257 (1992).

27. S. H. Chen and J. Teixeira, Phys. Rev. Lett. 57,2583 (1986).

28. J. E. Martin and B. J. Ackerson, Phys. Rev. A31, 1180 (1985).

29. D. Stauffer, Phys. Report, 54 , 1 (1979).

30. J. Rouch, A. Safouane, P. Tartaglia and S. H. Chen, J Chem. Phys. 90, 3756 (1990).

31. S. Magazu, D. Majolino, G. Maisano, F. Mallamace and N. Micali, Phys. Rev. A40, 2643 (1989).

32. Private communication from F. Mallamace (1991) 